\title{
Honours and (re)design of the curriculum: How to bridge the gap between honours and bachelor programmes
}

\author{
Annelies Riteco ${ }^{1 *}$, Irene de Kleyn ${ }^{2}$, Nicolle Lamerichs ${ }^{3}$
}

1. HU University of Applied Sciences Utrecht, The Netherlands, annelies.riteco@hu.nl

2. English Department of Teacher Education, HU University of Applied Sciences Utrecht, The Netherlands, irene.dekleyn@hu.nl

3. International Communication and Media, HU University of Applied Sciences Utrecht, The Netherlands, nicolle.lamerichs@hu.nl

*Corresponding author: annelies.riteco@hu.nl

Received: 15 February 2017; Accepted: 17 April 2017; Published: 9 May 2017

Keywords: talent development; personalized learning; co-creation; educational innovation; honours programmes; honours tracks; value creation

\begin{abstract}
Since 2016 the University of Applied Sciences Utrecht (Hogeschool Utrecht, HU) has been implementing a new HU Honours vision with value creation as the core element. Honours students realize value creation on several levels: personal growth and professional identity, and they deliver products or services that add value to professional practice and wider society. HU Honours education, which is accessible for all ambitious and talented students, offers five types of honours tracks. In order to bridge the gap between honours education and the bachelor programmes, new honours tracks are designed at the same time the regular courses are (re)designed. Honours is structurally embedded in the educational innovations and vice versa: honours is an excellent stimulus for curriculum (re)design because there are more innovative opportunities to set up learning and teaching in different ways. This integration of curriculum renewal and honours programme development has given a boost to new honours tracks and to talent development of all ambitious and talented students.
\end{abstract}

\section{Introduction}

HU University of Applied Sciences Utrecht (Hogeschool Utrecht, HU), as one of the hosts of the Utrecht Honours Conference Honours Futures, organized the preconference on June 1, 2016. The visit at the HU started with a short presentation of the renewed vision on honours and educational concept that has been implemented since 2016. 
From its inception HU Honours education has been accessible to all gifted and ambitious students. Different honours tracks are offered for different types of students: for students who want an in-depth grasp of a subject or a broader view of the matter, for students who prefer to learn more about a subject over an intensified or longer period using a highly structured or completely unstructured setup. Students follow honours programmes in addition to their bachelor programme and do not receive credits for this.

The focus of this paper will be the opportunities that arise to bring honours programmes closer to bachelor curricula while implementing curriculum design. The design or redesign of educational programmes is an excellent opportunity for the development of new honours tracks, especially the tracks which are in line with the regular courses (the so-called 'star courses' at HU are the regular courses providing students the option to participate in an honours track).

We start with a short introduction on the educational context of $\mathrm{HU}$, where a huge innovation is going on for all educational degrees, covering already more than 60 bachelor programmes in total. In the first section we will elaborate on the HU Honours vision and educational concept. Then, we will illustrate how the bachelor programme International Communication and Media implements honours in light of the redesign, to fit ideas of cocreation, personal development, and to truly create a community of excellence. In the last part, we will show how driven students are encouraged to further explore a topic of their interest from a regular course and to deepen and broaden their learning, thus exceeding the courses' regular attainment levels.

\section{Educational innovation at $\mathrm{HU}$}

In 2014 HU started with a large-scale curriculum renewal of its Bachelors and Masters programmes, including a redesign of its honours programme. During this curriculum development, the HU draws on its educational vision, based on the ideas of hundreds of HU lecturers and students. The HU's vision of education has been elaborated in the pamphlet Our world of tomorrow (HU University of Applied Sciences Utrecht, 2015).

Lifelong learning is the main principle: the HU wants to offer education for new and more experienced professionals. The HU vision of education has five principles: 1) lifelong learning, 2) education in co-creation with professional practice, 3 ) education based on practically-oriented research, 4) personalized learning as a framework for talent development (including honours), teaching methodology, and 5) blended learning which is a combination of face-to-face education, workplace learning, and online and team learning.

Based on the vision of education, fourteen design dimensions have been formulated that provide both direction and space to (re)design the education. Teams of lecturers are invited to give their own interpretation, shape and meaning to these dimensions. One of the dimensions is personalized learning. With personalized learning we mean that students devise their own learning process, in terms of pace, time, sequence, interest, content, goals and level. Honours is the ultimate form of personalized learning, because the students can decide themselves what topics they want to study, what kinds of projects they want to do, and what kind of issue they want to research. Personalized learning on content, goals and levels, beyond the curriculum borders of the bachelor programme, is possible for all ambitious HU students who want to get the best out of themselves and their study. 


\section{Honours renewed}

\subsection{Honours for all}

Honours education at the HU started in $\mathbf{2 0 0 9}$ with the Sirius Programme, the national incentive programme that gave a boost to excellence in the context of Dutch higher education.

From the beginning the guiding principle for the $\mathrm{HU}$ was that everyone has talents, so everyone can excel at the HU! Honours programmes should be accessible to all students at the $\mathrm{HU}$, not only for the high achievers. The HU has chosen to give the opportunity to all students to excel if they want and if they can. HU Honours is for the inquisitive students who are interested in the world around them and who want to have a larger impact on society.

However, these driven students differ a lot. That is the main reason for offering a broad palette of honours education. The HU offers five types of honours tracks, with different opportunities, in order to reach as many students as possible. The tracks are designed to reach different types of students depending on what they want to learn and achieve, how much time they can spend, what their learning style or preference is, and whether they want to deepen or broaden their knowledge and skills.

During the Sirius Programme, Honours Education occurred next to and usually outside regular education. It was a special project and despite the precondition that honours programmes should be accessible for all students, it was hard to reach many students and also teachers.

In 2014 the HU started with a major curriculum development in which honours was embedded and that has been very important to make honours education more accessible and to integrate it with other educational innovations. Honours is not a separate project anymore, but is connected to other educational and organizational developments.

This was also the moment to renew the honours concept. It is not a totally new approach of honours, but we have improved and changed some elements in our way of thinking. The HU already had a Star System that gave students a star for each honours achievement. In the renewed Honours concept the Star System will remain, but more opportunities for students to excel have been created.

\subsection{Value creation}

The core element in the renewed HU Honours vision is value creation, which means adding value in one or more of these four areas:

1. for themselves, to give meaning to their learning process, personal and professional development and career;

2. professional practice;

3. their specialist field;

4. society taken in the widest, most imaginative sense.

Value creation is derived from creatio, that means "creating something new". Honours students deliver products or services that add value to themselves, professional practice, their field or society. They want to make a difference in their environment, which can be 
very broad: a child at school, a parent, a patient, the director of an agency, a local organization, an interested party, an individual or a relevant target group.

As value creation is at the heart of honours education, an important function of honours is to educate honours students into being 'leading professionals in value creation'. But what makes a student a leading professional in value creation? A group of honours lecturers have made a general profile of this professional.

Figure 1: Profile of the leading professional in value creation

\begin{tabular}{ll|}
\hline Area of development & Qualities / competences \\
Learning: personal growth & Proactive personality \\
Value creation for professional practice & Innovative ability \\
Value creation for wider society & Integral thinking and acting \\
\hline
\end{tabular}

At the end of their study and honours programme students apply for a final honours assessment where they demonstrate how they have developed themselves into a professional who shows leadership qualities in value creation.

\subsection{Types of honours tracks}

As explained before, the precondition is that HU honours education has to be accessible to all motivated and ambitious students. As honours students have different needs and wishes, we offer five different kinds of honours tracks:

1. Pre-honours: a multi-disciplinary honours track in a series of ten evenings. The prehonours trajectory facilitates self-development and self-exploration in terms of identity and compassion within a framework of personal development, collaboration and regional project work. It is also an orientation programme in order to know the possibilities of honours. The programme was co-created together with a group of highly motivated second, third and fourth year honours students.

2. Complete, integrated honours programmes, for students who want to excel at the highest level. These programmes have been especially designed for students who want to work in a stimulating team with other honours students. Within the honours programmes students are able to choose their own path by creating their own projects. The time that students need to spend at an honours programme is similar to a minor, so 30 credits. But they do not get credits for honours, they can achieve stars for their honours achievements. Each honours activity represents an investment of 5 credits, 100 - 140 hours for each star. In a complete HP students do five different honours projects, assignments, activities, or courses, so they can obtain five stars and 
after obtaining five stars, they can apply for the final honours assessment in order to achieve the honours certificate.

3. Star courses. Honours students follow the regular bachelor course but they make extra assignments in line with the course or they do assignments at a more complex level. The predesigned star course provides a framework for further broadening or deepening in the topic of the regular course. Students can follow the course on two levels: regular and honours level. Each course of $5 \mathrm{EC}$ can lead to one star. Either lecturers who like to take on challenges with their students or the students themselves can take the initiative for this option.

4. Extra Honours - extra-curricular projects often with a direct cooperation with the work field. Lecturers often take the initiative for these kinds of projects or the stakeholders from professional field. It is about complex issues that can be solved by honours students or a team of honours students.

5. Personal track - students can work out their own idea, plan. This is the most free form of honours education. The students can get coaching or support from an honours coach. The student has to organize that. Every HU institute has many honours coaches and the students can consult them on demand. They write a proposal about their plan and if it has been approved, the student can start with his activities. The approval is a kind of "go", so the student knows that his plan has potential. If a plan is rejected, the student gets some suggestions on how to improve the proposal.

\subsection{Honours assessment}

How do we evaluate, assess the honours achievements, the contributions of honours students? We have developed our "Star System": students can acquire a star or a star certificate for his product, service, intervention or method with added value.

The stars are in line with the competences or the profile characteristics of the beginning excellent professional. The student can acquire a star for each demonstrated competence based on an honours achievement.

The five profile characteristics or stars are:

1. Reflective practitioner: the student possesses a high level of expertise, he has a good knowledge base and applies this in practice. And he reflects on professional action.

2. Innovation and dissemination: the student demonstrates the ability to innovate and makes a contribution to further development of professional practice.

3. International perspective: the student puts the profession and the activities in an international perspective and recognizes the consequences of different environments and cultures for the working fields.

4. Professional drive: the student is highly motivated to optimize his own qualities, makes conscious choices and has a clear vision on his profession.

5. Leadership qualities: the student leads and possesses the skills to plan and to collaborate with others. The student shows leadership. 
Before the renewal of honours education students had to achieve all five different stars, but it has been decided to skip that, because the characteristics are too different and in this way incomparable. Now the honours student can opt for the same star twice or three times. In order to create even more freedom for the students to choose their own path we have invented the so called 'free star'. Students can choose their own honours activity, have it assessed and gain the 'free star'. They devise their own star and can give it a name like 'entrepreneurship'. For the free star, the student has to demonstrate how he has created added value for the professional practice or society.

Once five stars have been acquired, students can apply for the final honours assessment during which they demonstrate that they have characteristics of a forward-thinking professional capable of value creation. Once they do so, they obtain an honours certificate. The existing star system and the new element of value creation come together in the final honours assessment for 'graduating with honours'.

\section{Integrating Honours in Curriculum Design}

We focus in this section on honours as connected to the redesign of the programme International Communication and Media (ICM) as a case study of how honours can be integrated within a wider educational landscape. This international bachelor programme has a truly international student population and staff from a wide range of countries both within and outside of Europe. The programme is currently undergoing a thorough redesign (20162020) to fit the needs of the professional field and categorize itself as a Creative Business bachelor.

This redesign is an opportunity to create a more thorough competence-based programme which focuses on higher impact learning. Courses are redeveloped to allow lecturers to teach in a blended way (Bersin \& Bersin, 2004; Vaughan, Cleveland-Innes \& Garrison, 2013), which bridges online and offline environments, and creates space for new classroom activities where students also participate in massive open online courses (MOOCs). Moodle is our primary tool in this redesign, to accommodate for large open courses, as well as smaller intimate ones.

In the redesign, ICM focuses on several key competences which align with honours well, such as lifelong learning and personal branding. Honours functions as both an innovator for existing courses and a space to creatively try out ideas. We find that honours has great merit as an excellence programme which is co-created. By emphasizing the individual student, the $\mathrm{HU}$ honours concept allows for innovative, personal ways of teaching.

The vision of ICM is emblematic of that of the HU in general. The merit of honours lies in the student's personal development, which is matched closely to ICM's vision at a micro level - a vision which emphasizes a personalized learning approach, co-creation, intercultural awareness, and the need to keep on learning to become an apt and forward-thinking professional. In this section we will illustrate how ICM implements honours in light of the redesign, to fit ideas of co-creation, and to truly create a community of excellence.

\subsection{Implementation of Honours at Programme Level}

To truly educate for the professional field of creative business, the honours research at ICM is envisioned both disciplinary and interdisciplinary. Students may want to deepen 
competences and subjects in their honours journey, but may also want to see this trajectory as an opportunity to go beyond the familiar disciplines (Wolfensberger, 2015, p. 157). The interdisciplinary aspects of honours are connected to the HU's institutional context, where honours students are encouraged to go beyond institutes and disciplines, and are invited to go on an "expedition" to find new qualities within themselves.

We encourage students to not only explain their honours project, but also focus on its international aspects, its relation to the ICM curriculum, and its value for the professional field. ICM is a truly interdisciplinary and intercultural programme, and we see honours as an opportunity to dive deeper into cultural competences. As an extra honours project, students can choose to for instance reflect more on their study abroad, and create a network based on their exchange. For this fixed project, we created a rubric which lists different competence levels for intercultural awareness and tests students based on a report and presentation.

\subsection{Walk the Talk}

In The Honours Experience, Van Eijl and Pilot (2016) describe that a growth mindset is a necessary quality for excellence - a mental disposition to seek new challenges and opportunities, instead of avoiding them (p. 17). Having such a mindset creates personal development. Both lecturers and students need to acquire that growth mindset, and keep learning and developing themselves. Co-creation, then, is integral in our vision of excellence. To truly excel at a University of Applied Sciences, undergraduates need to be professional and truly learn to collaborate and become leaders.

When discussing the practice of honours, recruitment cannot be overlooked. By connecting honours recruitment more actively to study coaching, we hope to identify suitable honours students sooner, and coach them in their personal learning paths. In their study Van Eijl and Pilot (2016, p. 81) emphasize that creating a culture of excellence is fundamental to identifying and developing excellence. This culture also implies that study coaches have to be able to spot excellence, and actively recruit students (ibid., p. 89). In our previous curriculum design, coaching focused on students who were delayed, or who had special needs. This has the danger that excellence is not recognized or valued as much, when in fact it can be nurtured and enrich students' education tremendously.

In our current redesign, much attention is paid to personalized learning trajectories of all students, and to the excellence of students and staff. Our vision for redesign is our explicit ambition to create an honours community which is driven by students, and coached by inspiring staff members. Like all HU institutes, lecturers are inspired to "walk the talk" and engage students by giving the right example as role-models, who also keep learning themselves. This idea of co-creating honours as a dialogue of students and staff is integral to the ICM vision. This also implies that excellence within staff should be encouraged. As a University of Applied Sciences, we may reward this differently than many other modern universities who emphasize research.

In our vision of honours, we see honours as aligned to didactical methods (e.g. coaching, teaching), and as added value to the professional field. Co-creation is central in this notion, both connected to the professional field as well as to didactics. Our idea of co-creation is 
perhaps best visualized when considering a recent COIL (collaborative online international learning) course developed with an Australian partner university, RMIT College of Business, Melbourne. COIL is best described as: '...courses, projects and other "virtual exchange" or 'telecollaboration' activities that bring university students and academics together across borders. Integrating it into the curriculum enhances the intercultural competence of students who might not otherwise have the opportunity to study abroad' (European Coil Conference, 2017).

Connecting honours with COIL has proven valuable at our programme, where it is considered an opportunity to educate intercultural competence at a high level. Lecturers give much room for students to follow their interests. While a rudimentary rubric was designed, and some assignments were described, developers left much space for students to initiate their own projects with their Australian peers. As we are now in the pilot phase, the learning outcomes have not been evaluated yet, but a constant dialogue with students and our foreign partners was crucial in the development and vision overall.

\subsection{Innovation and Play}

Within the curriculum, we also use honours as innovator to experiment with new didactic visions, which do not only include co-creation, but also more interactive learning activities. Specifically, we want to use honours education as a way of experimenting with more blended activities, including games and play in education.

For education, gaming has clear merits. Digital and non-digital games function as valuable vessels for identity formation and social grouping (Pearce, 2009). Games are effective educational tools, because they provide a space where conventions of the everyday world cease to exist. Games suspend disbelief, and also create immersion within educational activities.

In education, temporarily reforming the group through play can be a powerful tool. Roleplaying and other activities can create a more dynamic, engaging and emphatic way of learning activities, skills and knowledge. Through play, students can also experiment with different attitudes, making all of these elements suitable for competence-based learning. Within honours, games can be a way to playfully master learning goals and key competences. For instance, one of the competences of the excellent professional is "leadership", which can easily be activated within the context of gaming. As a game design method, we can create a state of play or simulation which relies on complex, social roleplaying. This simulation can be developed into more full-fledged games of several hours, such as live-action events where management strategies are played out in the flesh. Here, the safe space of the game can give students a feeling of security, and experimentation to try out different leadership strategies.

Casting our students in the role of playful learners is something that we value at ICM, and at HU level in general. This resonates with our honours vision, which is envisioned like a heroic journey (e.g., Van Eijl \& Pilot, 2016) or expedition. Overall, honours functions as an innovator at ICM. It tests our competences, but also allows students to go beyond our fixed programme. We see honours as an opportunity to implement new didactic methods, and to truly co-create content with our students. The honours programme is as much student- 
driven as it is facilitated by staff members. We leave much room for students to find and create their own projects or courses which meet their needs and help them flourish as professionals.

\section{Honours as an option in regular courses}

In this section we will illustrate how motivated students, willing to add value to themselves personally and professionally, the work place or wider society, can choose for an extra honours option in a regular bachelor course. The English Department for Teacher Education at HU currently provide a series of star courses in the Methodology curriculum. Based on regular Bachelor Methodology courses and learning goals, keen students have the opportunity to deepen and broaden their learning, thus exceeding the courses' regular attainment levels.

In the Methodology courses students study concepts such as Communicative Language teaching and learning, language skills, collaborative work, engaging activities, digital learning tools, differentiation, Task-Based Instruction, use of target language, dual language education, formative and summative assessment and curriculum design. Driven students are encouraged to further explore a topic of their interest and become competent with its practical application, and in doing so, earn Honours stars according to the HU Star system: reflective practitioner, innovation and dissemination or professional drive. The predesigned star courses provide a framework, yet allow students to determine the success criteria of their final product. The outcome is noteworthy as is demonstrated by the following two examples:

1. A driven part-time trainee English teacher noted that, although her vocational internship college claimed to provide an authentic learning environment for students, their summative assessments were far from it. She decided to enrol in the Methodology star course with the objective to create authentic and relevant learning opportunities with the focus on natural learning processes and general practical application that would motivate the vocational students. After a process of research, creation, evaluation and improvement, her newly developed authentic summative assessments have been embedded in the curriculum at the college which resulted in two Honours stars for reflective practitioner and innovation and dissemination. Boosted by this achievement the student has decided to continue with the Honours Programme and is presently doing research into early school leaving at vocational colleges in order to establish a bespoke support plan.

2. An enthusiastic full-time English student was eager to further explore the use of digital learning tools in order to enhance teaching and learning in the Modern Foreign Language classroom. Her aim was to inspire teachers and fellow students to apply innovative online digital learning tools in their teaching practice. Her research into the added value of engaging ICT tools on learning outcomes has resulted in the creation of todaysteachingtools.com, a website with categorised lists of trialled online digital learning tools, the publication of weekly blogs and two Honours stars for innovation and dissemination. Thanks to her social media platform the project has evolved ever since with her regularly conducting workshops on digital learning tools and creating digital learning environments at schools and companies for which 
she has been rewarded with two additional stars; professional drive and reflective practitioner.

Star courses grant motivated students access to the Honours Programme and allow them to be self-initiating and creative within a framework. The aforementioned projects demonstrate the endless opportunities that may arise when students, once aware of their possible impact on their surroundings, are willing to add value to themselves personally, professionally, to the workplace and the wider society. Stars creating stars.

\section{Concluding remarks}

In this paper we have elaborated on the HU Honours vision and educational concept, with value creation as the core element. Despite the precondition that honours programmes should be accessible for all ambitious and talented students, it was hard to reach many students, probably because of the gap between honours programmes and the regular bachelor curriculum. In order to bridge this gap, new honours tracks have been designed at the same time the regular courses were (re)designed. Honours is structurally embedded in the curriculum innovations and vice versa: honours is a perfect stimulus for curriculum (re)design because of its innovative character. We have shown this for one bachelor programme in particular: International Communication and Media. We have also illustrated how students can choose for an extra Honours option in a regular bachelor course, another opportunity that arises to bring honours programmes closer to bachelor curricula.

\section{References}

Bersin, J. (2004). The Blended Learning Book. San Francisco: John Wiley and Sons.

European Coil Conference (2017). About COIL. Retrieved from: https://eurocoilcon.nl/

HU University of Applied Sciences Utrecht. (2015). Onze wereld van morgen. Visie op Onderwijs. Retrieved from: http://www.huontwikkelt.nl/onderwijs-onderzoek/huonderwijsvisie/

Huizinga, J. (1970). Homo Ludens: A Study of the Play Element in Culture. London: Maurice Temple Smith Ltd.

Pearce, C. (2009). Communities of Play: Emergent Cultures in Multiplayer Games and Virtual Worlds. Cambridge; London: MIT Press.

Vaughan, N., Cleveland-Innes, M., \& Garrison, R. (2013). Teaching in Blended Learning Environments: Creating and Sustaining Communities of Inquiry. Edmonton: AU Press.

Van Eijl, P. \& Pilot, A. (2016). The Honours Experience. Rotterdam: Hogeschool Rotterdam Uitgeverij. 
Page 11 of 11

Wolfensberger, M. (2015). Talent Development in European Higher Education: Honours Programmes in the Benelux, Nordic and German-Speaking Countries. Heidelberg; New York;

Dordrecht; London: Springer. http://link.springer.com/book/10.1007\%2F978-3-319-12919-8 